(azathioprine, cyclophosphamide, mycophenolate mofetil), independently by the ILD pattern and $21(70 \%)$ low dosage of steroids.

After a median period of 23.5 months (range 11-111), 7 patients developed an ANCA associated vasculitis, while other 3 developed other rheumatic diseases. Finally, when compared with IPF, ILD-MPO patients had a better survival $(81.2 \% \pm 0.9$ vs $54.7 \pm 0.7$ for ILD-MPO and IPF, respectively; $p=0.045)$

Conclusion: ILD positive for anti-MPO antibodies are still a not definite condition. We need larger population to identify possible markers for the evolution in an ANCA associated vasculitis, to define the prognosis of disease and the better therapeutic approach.

References: :

[1] Mohammad AJ, et al. Pulmonary Involvement in Antineutrophil Cytoplasmic Antibodies (ANCA)-associated Vasculitis: The Influence of ANCA Subtype. J Rheumatol. 2017;44:1458-67

Disclosure of Interests: None declared

DOI: 10.1136/annrheumdis-2020-eular.4614

\section{AB0529 \\ TEMPORAL ARTERY ULTRASOUND (TAUS) IS A RELIABLE TECHNIQUE TO RULE OUT GCA EVEN IN THE LEARNING PHASE}

\section{A. Sidhu ${ }^{1}$, A. Bharadwaj ${ }^{1}$, A. Nandagudi . ${ }^{1}$ Basildon University Hospital,} Rheumatology, Basildon, United Kingdom

Background: Giant cell arteritis (GCA) is an emergency. The initial treatment with high dose glucocorticoids (GC) is often started on clinical suspicion without waiting for Temporal artery biopsy (TAB) results, which can take days to be available. TAUS is a simple, non-invasive test which is readily available. However, like any other ultrasound, it is also operator dependent. A positive halo sign is the most specific abnormality seen on TAUS in GCA patients. The percentage of false positive TAUS in GCA diagnosis is low (1), but it can result in over diagnosis and unnecessary exposure to high dose GC in elderly population.

Objectives: We looked at the reliability of TAUS in ruling out GCA after it was introduced within our rheumatology department one year ago.

Methods: We adopted the quality improvement methodology for assessment. Retrospective data of suspected GCA patients was collected over the last two years. TAUS was introduced regularly to the investigative plan after eleven months. Two Rheumatology consultants were trained in TAUS. Results were compared before and after the introduction of ultrasound as a diagnostic tool. In collecting the data, our main focus for documentation was based on clinical symptoms, TAUS and TAB results. We aimed to increase the awareness of appropriate GCA referrals among the primary and secondary care with the support of teaching sessions.

Results: From January 2018 to November 2019, 101 patients were referred to rheumatology with suspected GCA. Median age of our cohort was 72 years with male to female ratio of 1:3. 35 patients were referred in the first 11 months out of which, $10(28.6 \%)$ were diagnosed with GCA. TAUS and TAB was done in $20 \%$ and $49 \%$ of patients respectively. 66 patients were referred in the next 12 months after TAUS was introduced. Out of 66,14 patients $(21.2 \%)$ were diagnosed as GCA. TAUS and TAB were done in $82 \%$ and $38 \%$ of the patients respectively. As listed in table 1, only 1 patient was found to have positive TAB after a negative TAUS (false negative). All of patients with positive TAUS were treated as GCA on the basis of clinical grounds, irrespective of TAB results. Despite the regular use of TAUS as a diagnostic tool in the second phase, there is a higher percentage of patients $(78.8 \%)$ in which GCA was ruled out.

\begin{tabular}{lcc}
\hline TAUS introduction & $\begin{array}{c}\text { Before regular TAUS } \\
\text { (Jan 2018 - Nov 2018) }\end{array}$ & $\begin{array}{c}\text { After regular TAUS } \\
\text { (Dec 2018 - Nov 2019) }\end{array}$ \\
\hline Patients referred & 35 & 66 \\
GCA & $10(28.6 \%)$ & $14(21.2 \%)$ \\
Not GCA & $25(71.4 \%)$ & $52(78.8 \%)$ \\
TAUS done in & $20 \%$ & $82 \%$ \\
TAB done in & $49 \%$ & $38 \%$ \\
TAUS -ve and TAB +ve & 0 & 1 \\
TAUS +ve and TAB -ve/not done & 2 & 8 \\
\hline
\end{tabular}

Conclusion: After the routine introduction of TAUS, the percentage of patients diagnosed with GCA has declined and clinicians have been able to exclude suspected GCA diagnosis in a larger proportion of patients referred. This is noteworthy as our Rheumatologists are still in the learning phases of determining the significance of utility of TAUS. There is only a small decline in TAB frequency, which is expected to go down further in the coming years. We also noticed that the number of patients referred has almost doubled. This might be due to better education and awareness at the primary and secondary care level which was done as part of the project.

References:

[1] Fernández E, Monjo I, Bonilla G, et al OP0210 FALSE POSITIVES OF ULTRASOUND IN GIANT CELL ARTERITIS. SOME DISEASES CAN ALSO HAVE HALO SIGN Annals of the Rheumatic Diseases 2019;78:181

Disclosure of Interests: None declared

DOI: 10.1136/annrheumdis-2020-eular.6540

\section{AB0530 1 CHARACTERISTICS AND MEDIUM-TERM OUTCOMES OF TAKAYASU ARTERITIS-RELATED RENAL ARTERY STENOSIS: ANALYSIS OF A LARGE CHINESE COHORT}

Y. Sun ${ }^{1}$, X. Dai ${ }^{1}$, P. LV ${ }^{2}$, Z. Dong ${ }^{3}$, J. Lin ${ }^{2}$, L. Jiang ${ }^{1} .{ }^{1}$ Department of Rheumatology, Zhongshan Hospital, Fudan University, Shanghai, China; ${ }^{2}$ Department of Radiology, Zhongshan Hospital, Fudan University, Shanghai, China; ${ }^{3}$ Department of Vascular Surgery, Zhongshan Hospital, Fudan University, Shanghai, China

Background: The incidence of renal artery stenosis in Takayasu arteritis (TA) was $20 \%-60 \%$ according to previous reports. The specific characteristics of patients with TA-related renal artery stenosis and the effect of revascularization procedures on prognosis have not been fully investigated.

Objectives: To investigate the characteristics of patients with TA-related renal artery stenosis and identify the predictors of medium-term adverse outcomes. Methods: Data for 567 patients registered in a large prospective observational cohort-the East China Takayasu arteritis cohort-up to April 30, 2019, were retrospectively analyzed.

Results: Renal artery stenosis was confirmed in 172/567 (30.34\%) patients with left renal artery involvement seen in 73/172 (42.44\%) patients. Renal insufficiency at presentation ( $\mathrm{HR}=2.37,95 \% \mathrm{Cl}: 1.76-15.83, \mathrm{p}=0.03)$, bilateral renal artery involvement $(\mathrm{HR}=6.95,95 \% \mathrm{Cl}: 1.18-21.55, \mathrm{p}=0.01)$, and severe $(>75 \%)$ stenosis $(\mathrm{HR}=4.75,95 \% \mathrm{Cl} 1.08-11.33, \mathrm{p}=0.05)$ were predictors of adverse outcomes. Revascularization was performed for $46 / 172(26.74 \%)$ patients. Patients without preoperative treatment had higher rate of restenosis $(44.44 \%$ vs. $15.79 \%$, $p<0.01)$ and hypertension deterioration $(25.93 \%$ vs. $10.53 \%, p<0.01)$ after the procedure. Non-receipt of preoperative treatment $(\mathrm{HR}=6.5,95 \% \mathrm{Cl}: 1.77-$ 32.98, $\mathrm{p}=0.04)$ and active disease at revascularization $(\mathrm{HR}=4.21,95 \% \mathrm{Cl}$ 2.01-21.44, $p=0.04$ ) were independent predictors of adverse outcomes after revascularization

Conclusion: Patients with uncontrolled or worsening hypertension or/and renal function may benefit from revascularization. Those who have received preoperative treatment may have more favorable revascularization outcomes. Prognosis appears to be poorer for patients with renal insufficiency at presentation, bilateral artery involvement, and severe stenosis.

References:

[1] Watanabe Y, Miyata T, Tanemoto K. Current clinical features of new patients with Takayasu arteritis observed from a cross-country research in Japan: age and sex specificity. Circulation 2015; 132: 1701-9.

[2] Peng $M$, Jiang $X J$, Dong $H$, et al. Etiology of renal artery stenosis in 2047 patients: a single-center retrospective analysis during a 15-year period in China. J Hum Hypertens 2016; 30: 124-8

Disclosure of Interests: None declared

DOI: 10.1136/annrheumdis-2020-eular.3427

$\mathrm{AB} 0531$

GOLIMUMAB IN THE TREATMENT OF SEVERE AND/ OR REFRACTORY VASCULO-BEHÇET'S DISEASE: A SINGLE-CENTRE EXPERIENCE IN CHINA

L. Sun ${ }^{1}$, J. Liu ${ }^{1}$, W. Zheng ${ }^{1} .{ }^{1}$ Peking Union Medical College Hospital,

Department of Rheumatology, Beijing, China

Background: Vascular involvement is one of the leading causes of mortality and morbidity in Behcet's Disease (BD) ${ }^{1}$. Surgical treatments are difficult for Vaculo-BD (VBD) patients due to the high risk of serious postoperative complications without effective and promptly perioperative immunotherapy ${ }^{2,3}$. Anti-tumor necrosis factor alpha (TNF-a) therapy has been reported as a potential treatment in severe VBD, e.g. infliximab (IFX) and adalimumab (ADA). However, only few case reports are available regarding the fully humanized monoclonal antibody to TNFa, golimumab (GOL), in the management of $\mathrm{VBD}^{4}$.

Objectives: The objective of this study was to report the efficacy and safety of GOL for the treatment of severe and/or refractory VBD.

Methods: We retrospectively analyzed the efficacy and safety profile of patients with severe and/or refractory VBD treated with GOL in our medical center between 2018 to 2020 . 